\title{
MODE OF UMBILICAL CORD INSERTION AND NEONATAL WEIGHT,
} AND SOME PLACENTAL FACTORS

\section{Tsegah M. Korantema *1, Asante DuBois ${ }^{2}$.}

${ }^{* 1}$ Department of Biomedical Sciences, School of Allied Health Sciences, College of Health and Allied Sciences, University of Cape Coast, Cape Coast, Ghana.

${ }^{2}$ Department of Forensic Sciences, School of Biological Sciences, College of Agriculture and Natural Sciences, University of Cape Coast, Cape Coast, Ghana.

\section{ABSTRACT}

Background: Birth weight is an important determinant of health of a child, with low birth weight associated with the occurrence of many chronic diseases in adult life.

Aim: The objective of this study is to determine the correlation between mode of insertion of umbilical cord and neonatal weight and some placental variables.

Methods: Forty placentas were obtained from the Cape Coast Teaching Hospital in the Central Region. Umbilical cord length was measured and its position on the placenta noted and recorded. The placentas were trimmed and measurements of placental weight, diameter, and thickness were measured using Olympic smart scale and anthropometric measure respectively and then the volume were also recorded.

Results: Three forms of umbilical cord insertion on the placentas were observed: central, eccentric and marginal. The result obtained shows that $57.5 \%$ of the umbilical cord insertions were eccentric, central cord insertions were $11(27.5 \%)$ and marginal insertions were $6(15 \%)$.

Conclusion: The results were analyzed using Pearson's Chi-square and showed that the umbilical cord inserts more eccentrically and that mode of umbilical cord insertion has no association with the placental variables and birth weight looked at and thus mode of cord insertion may not significantly influence birth weight of the neonate. There was however a positive association between placental index and umbilical cord length $(P<0.05)$. KEY WORDS: Umbilical Cord, Placental Variables, Neonatal Weight, Umbilical Cord Insertion.

Address for Correspondence: Mrs. Tsegah M. Korantema, Department of Biomedical Sciences, School of Allied Health Sciences, College of Health and Allied Sciences, University of Cape Coast Cape Coast, Ghana. E-Mail: ktsegah@ucc.edu.gh

Access this Article online

Quick Response code

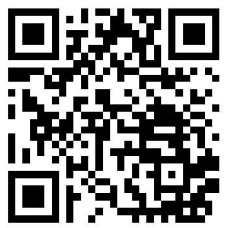

DOI: $10.16965 /$ ijar.2018.248

$$
\text { Journal Information }
$$

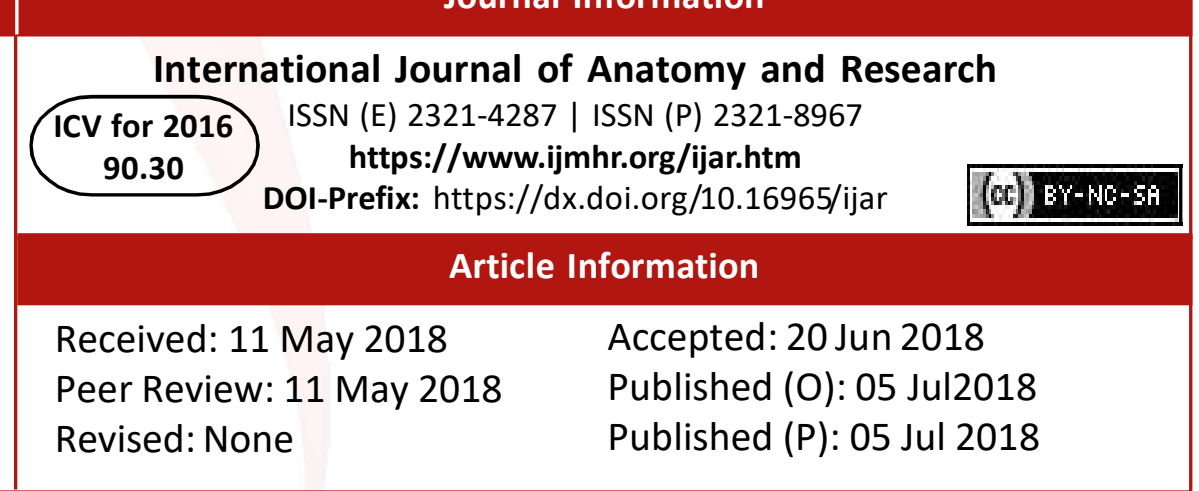

\section{INTRODUCTION}

The mode of insertion of the umbilical cord on the placenta is essential for the growth of the foetus and its development in the uterus of the mother [1]. The foetus derives its nutrient and oxygen as well as other necessary immune cells from the mother through the umbilical cord that connect the foetus to the placenta [2].
Birth weight is an important determinant of child health [3].

Low birth weight is defined as baby less than 2.5 kilograms ( 5.5 pounds) at birth, with premature birth (preterm) being the common cause of such low birth weight babies. Low birth weight (LBW) is responsible for a very significant proportion of morbidity and mortality in childhood, 
but children born with average birth weights are reported to do well even under relatively adverse environmental conditions [4]. Low birth weight is now also incriminated in occurrence of many a chronic disease in adult life [5]. LBW infants are 40 times more likely to die in their first month of life than normal birth weight infants. LBW infants are also twice as likely as other infants to exhibit health problems and serious developmental delays during childhood [5].

Several factors contribute to low birth weight which include teenage pregnancies, unwanted or unintended pregnancies, lack of prenatal care, malnutrition during pregnancy leading to poor maternal weight gain, maternal smoking and alcoholism and other drugs used during pregnancy may also contribute to LBW [6]. Maternal age, health, foetal infection, ethnicity, multiple births, genetic makeup, obstetric history, and a variety of genetic and metabolic disorders can also contribute to low birth weight [4]. Women below 20 years and over 40 years of age are more likely to have a low birth weight baby [5]. Birth weight is inversely associated with risk of adult cardiovascular disease, and evidence exist that foetal adaptations to challenges in the intrauterine environment may adversely affect long-term cardiovascular health [4]. A disproportionately large placenta (with a ratio greater than $1: 6)$ relative to birth weight is associated with increased risk of cardiovascular disease and death [5]. Findings by Bloomberg (2009), suggests that placenta function is important in the association of intrauterine factors with cardiovascular disease later in life.

During gestation, the fertilized ovum goes through several developmental stages. By the fifth week, the yolk sac, duct, allantois, and umbilical vessels are restricted to the region of the umbilical ring [1]. The placenta is fully developed by the third month of pregnancy [1]. The placenta shape is roughly spherical and is formed by the inner portion of the chorion of the embryo and a portion of the mother's endometrium [2]. It allows the foetus and the mother to exchange gas, nutrient and waste, and secretes hormones necessary to maintain pregnancy [2]. A layer of maternal epithelial cells in the villi and of endothelial cells in the foetal capillaries separates the maternal and foetal blood [7]. Waste products move from fetal blood through foetal capillaries across the maternal epithelium into the maternal blood, and nutrient, hormones and growth factors move in the opposite direction [7] and connected to the placenta is the umbilical cord. The umbilical cord forms as the yolk sac shrinks and the amnion expands to envelop the embryo.

A fully formed umbilical cord is about 1 to $2 \mathrm{~cm}$ ( 0.5 to $1 \mathrm{in}$ ) in diameter and approximately 55 $\mathrm{cm}(2 \mathrm{ft}$ ) long [8]. The umbilical cord contains two arteries which carry oxygen- poor blood and metabolic waste towards the placenta, and a vein that carries oxygen-rich blood and nutrients from the placenta to the embryo. These vessels are surrounded by embryonic connective tissue called mucoid connective tissue otherwise known as Wharton's jelly and the umbilical cord is inserted on the placenta in different modes. The cord is more eccentrically situated on the chorionic plate but it may vary from a midcentral or central insertion to a marginal attachment [9]. A marginally inserted umbilical cord results in a battledore placenta [10]. Some reports have associated a marginal attachment of the umbilical cord to the placenta with a variety of anomalies in the course of pregnancy and neonatal development. These include premature labour, neonatal asphyxia, abortions [11], malformed infants [12], foetal growth failure and stillbirth [13]. Although the umbilical vessels typically remain close to each other until the placenta is reached, they occasionally separate some distance from the placenta, giving rise to a furcated placenta [10]. This mode of insertion is known as insertio funiculi furcate [14]. The vessels are believed to lose their protective covering of Wharton's jelly before reaching the placental surface [9].

The clinical significance of these conditions resides in the fact that exposed vessels are highly susceptible to damage. Sometimes there is velamentous insertion which means that the veins, unprotected by Wharton's jelly, traverse the membranes before they come together into the umbilical cord or inserts on the chorioamniotic membranes rather than on the placental mass. Complications of umbilical cord have been implicated in the cause of intrauterine foetal deaths [8]. Hence, does the mode of 
insertion of the umbilical cord on the placenta affect placental variables such weight and volume and thus birth weight?

\section{METHODOLOGY}

The research was carried out in the Cape Coast Teaching Hospital, located near Abura a suburb in the Cape Coast Metropolis in the Central Region of Ghana. Placentas from babies delivered by spontaneous vaginal delivery by healthy mothers were collected from the delivery suite of the hospital and used immediately for the study. Consents of mothers of the neonates whose placentas were used were sought prior to the study.

The placentas together with their umbilical cords were collected trimmed, and examined to ensure that there were no missing tissue suggestive of retained placental tissue in the mother and the umbilical cord was severed from the placenta at the point of insertion. The length of the umbilical cord was then measured with an anthropometric measure. The readings were recorded to the nearest $1 \mathrm{~mm}$. The diameter of the placenta was measured with an anthropometric measure by considering the broadest diameter, three readings were taken for each placenta with approximately equal diameter and the average value was calculated. The weight of the placenta was measured using the Olympic smart scale was recorded to the nearest 10 grams. The weighing was completed within fifteen minutes of delivery to preclude drastic changes in volumetric composition [15].

The volume of each placenta was determined by liquid displacement as described by Mayhew [16] and recorded to the nearest $5 \mathrm{~cm}^{3}$. Each placenta was placed on a flat board and then sectioned into equal quadrants (Figure2) and choosing a random starting point, a tissue was sampled full-depth (from chorionic plate to basal plate) from each quadrant concentrically and from the periphery to the center. The random starting point means that all areas had the same chance to contribute to the sample [17]. The thickness (full-depth) of the placenta was measured with a rule from the surfaces of the four quadrants and the average of the reading (to the nearest $0.01 \mathrm{~cm}$ ) was calculated to designate the thickness of the placenta.
The neonates were weighed to the nearest 10grams (g) using an Olympic Smart weighing scale and recorded as birth weight (BW). The sex of the neonate (NS) was noted and recorded as males (M) and females (F). Maternal age (MA) and gestational age (GA) were abstracted from the labour ward records of the mother.

Fig. 1: Foetal surface of placenta showing umbilical cord (UC) point of insertion (P) which is centric [18].

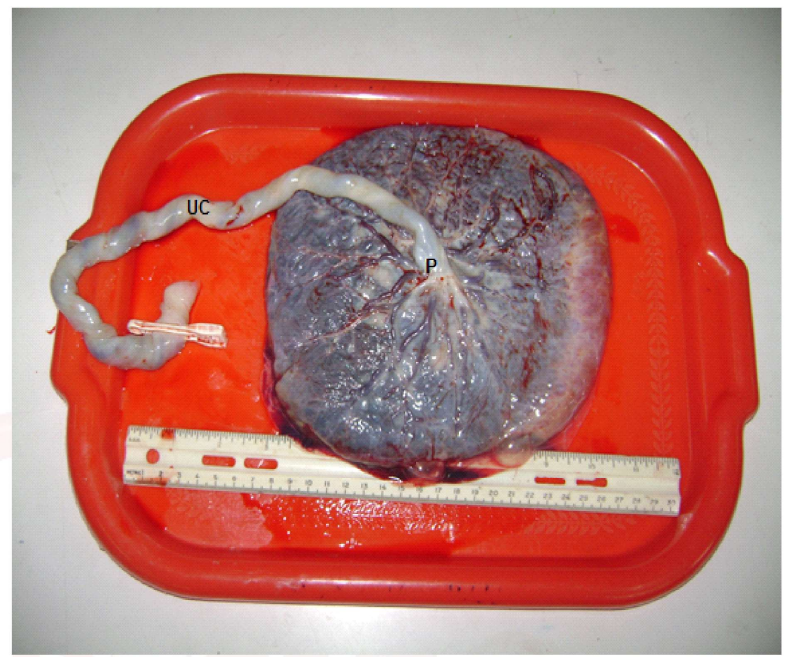

Fig. 2: A placenta cut into quadrants to measure thickness.

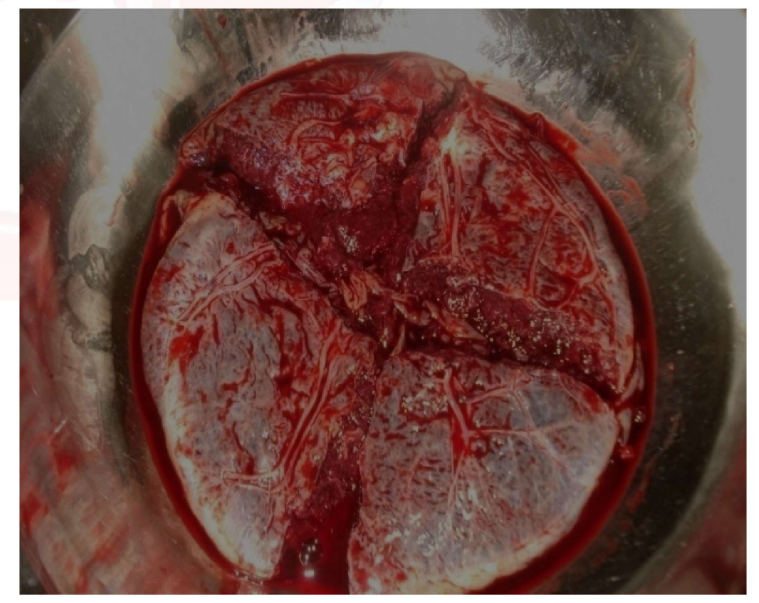

Data was analyzed using; SPSS version 17, MINITAB version 16 and Microsoft Excel 2007, descriptive analysis was made by using ANOVA to test for the magnitude and significance of any relation between the mode of umbilical cord insertion and the placental variables and neonatal weight that were recorded.

\section{RESULTS}

Out of 40 placentas, 31 (77.5\%) were delivered after a gestational period between 38 and 40 weeks and 9 (22.5\%) were between 33 and 37 weeks of gestational period. The maternal ages ranged between 22 and 37 years (Table 1). 
Table 1: Neonatal and placental variables and maternal age (with minimum and maximum values).

Standard Error of Means $($ Mean \pm SEM)

\begin{tabular}{|l|c|c|c|c|}
\hline \multicolumn{1}{|c|}{ Variables } & Mean & S.E & Minimum & Maximum \\
\hline Neonatal weight (g) & 3172.9 & 107.96 & 1485 & 5200 \\
\hline Placental weight (g) & 452.1 & 13.2 & 250 & 630 \\
\hline Placental index (g/g) & 0.15 & 0.01 & 0.09 & 0.28 \\
\hline Placental volume (cm ${ }^{3}$ ) & 409.83 & 14.6 & 230 & 600 \\
\hline Placental thickness (cm) & 1.96 & 0.06 & 1.2 & 2.5 \\
\hline Placental diameter $(\mathrm{cm})$ & 19.48 & 0.31 & 16 & 24 \\
\hline Cord length (cm) & 41.43 & 1.61 & 21 & 81 \\
\hline Maternal age (yrs) & 28.85 & 0.63 & 22 & 37 \\
\hline Gestational age (wks) & 37.43 & 0.28 & 33 & 40 \\
\hline
\end{tabular}

Table 2: Basic data on neonatal weight, placental variables, maternal age and gestational age in relation to neonatal sex (male and female) with standard error (SE).

\begin{tabular}{|c|c|c|c|c|c|c|c|c|c|}
\hline & BW & PW & PI & PV & PT & PD & CL & MA & GA \\
\hline FEMALE: MEAN & 3145.4 & 451.3 & 0.1 & 398.5 & 1.9 & 19.4 & 39.3 & 29.1 & 37.2 \\
\hline N & 19 & 19 & 19 & 19 & 19 & 19 & 19 & 19 & 19 \\
\hline SE & 180.8 & 15.9 & 0 & 19 & 0.1 & 0.5 & 1.8 & 1 & 0.5 \\
\hline MALE: MEAN & 3197.7 & 452.8 & 0.1 & 420 & 1.9 & 19.4 & 43.2 & 28.6 & 37.5 \\
\hline N & 21 & 21 & 21 & 21 & 21 & 21 & 21 & 21 & 21 \\
\hline SE & 128.8 & 20.9 & 0 & 22.1 & 0.1 & 0.4 & 2.6 & 0.8 & 0.3 \\
\hline
\end{tabular}

Fig. 3: A graph showing correlation of placental thickness, PT $(\mathrm{cm})$ and placental index, $\mathrm{PI}(\mathrm{g} / \mathrm{g}) . \quad(r=-0.19, \mathrm{p}<0.907)$.

Table 3: Neonatal weight and placental weight in relation to umbilical cord position with frequency $(n)$ of cord insertion.

\section{S.E $=$ Standard Error}

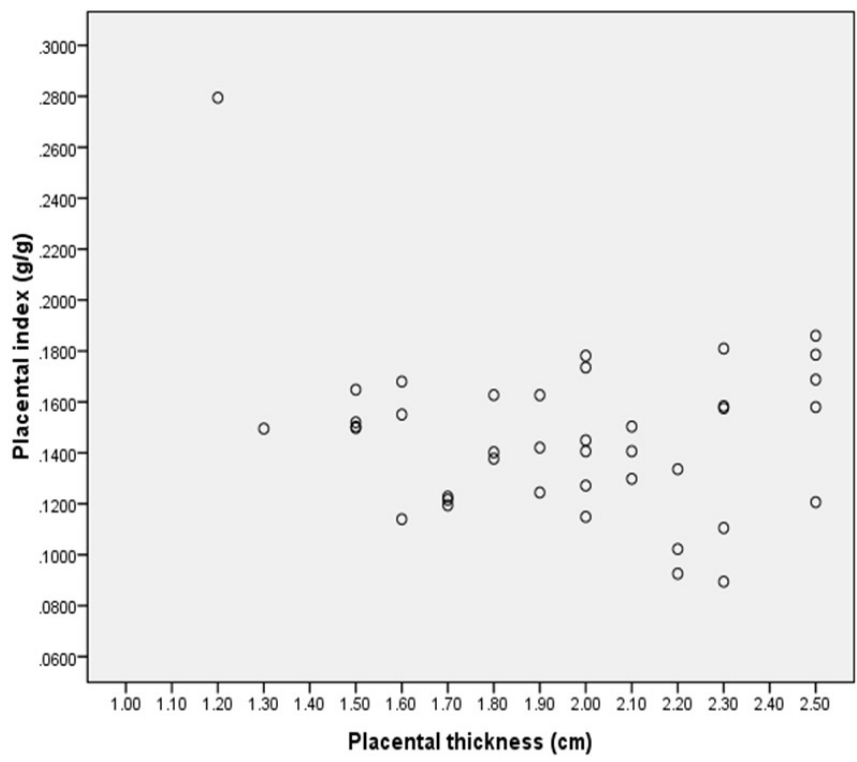

\begin{tabular}{|c|c|c|c||c|c|c|}
\hline $\begin{array}{c}\text { Cord } \\
\text { Insertion }\end{array}$ & \multicolumn{3}{|c|}{ Neonatal weight in grams (S.E) } & \multicolumn{3}{|c|}{ Placental weight in grams } \\
\hline & Female & Male & Total & Female & Male & Total \\
\hline \multirow{4}{*}{ Central } & $(n=6)$ & $(n=5)$ & $(n=11)$ & $(n=6)$ & $(n=5)$ & $(n=11)$ \\
& 3530.00 & 3012.00 & 3294.55 & 468.33 & 408.80 & 441.27 \\
& $(446.52)$ & $(136.65)$ & $(253.82)$ & $(28.48)$ & $(35.54)$ & $(23.21)$ \\
\hline \multirow{4}{*}{ Eccentric } & $(n=10)$ & $(n=13)$ & $(n=23)$ & $(n=10)$ & $(n=13)$ & $(n=23)$ \\
& 2882.30 & 3289.85 & 3112.65 & 455.50 & 468.08 & 462.61 \\
& $(189.07)$ & $(191.92)$ & $(139.89)$ & $(23.39)$ & $(22.54)$ & $(15.99)$ \\
\hline \multirow{4}{*}{ Marginal } & $(n=3)$ & $(n=3)$ & $(n=6)$ & $(n=3)$ & $(n=3)$ & $(n=6)$ \\
& 3253.33 & 3108.33 & 3180.83 & 403.33 & 460.00 & 431.67 \\
& $(249.69)$ & $(300.75)$ & $(177.79)$ & $(29.059)$ & $(105.63)$ & $(50.61)$ \\
\hline \multirow{4}{*}{ Total } & $(n=19)$ & $(n=21)$ & $(n=40)$ & $(n=19)$ & $(n=21)$ & $(n=40)$ \\
& 3145.42 & 3197.76 & 3172.9 & 451.32 & 452.81 & 452.1 \\
& 180.86 & 128.82 & 107.96 & 15.99 & 20.95 & 13.2 \\
\hline
\end{tabular}


Twenty one of the placentas obtained were from the delivery of male neonates and 19 from female neonates (Table 2).

The mean value obtained for each set of variables studied is presented in table 1 . The value for neonatal or birth weight (BW) was 3172.90g and (SE 107.97), placental weight (PW) was $474 \mathrm{~g}$ (SE 14.23), and that for placental index (PI) was 0.15 (SE 0.01). The value for placental volume (PV) obtained was $409.83 \mathrm{~cm}^{3}$ (SE 14.60), whilst that for placental diameter (PD) was $19.48 \mathrm{~cm}$ (SE 0.31). Placental thickness (PT) was $1.96 \mathrm{~cm}$ (SE 0.06), maternal age (MA) and gestational age were 28.85 years (SE 0.63), and 37.43 weeks (SE 0.28 ) respectively.

Table 2 provides information on male and female neonates with respect to neonatal weight and placental variables. Mean birth weight (BW) was 3,197.76 g for male neonates and 3145.42g for female neonates. Result from table 2 shows no significant difference between gestational ages for male and female neonates, 37.57 weeks (SE 0.34) and 37.26 weeks (SE 0.47) respectively. Placental index (PI) for male neonates was 0.14 (SE 0.01) and 0.15 (SE 0.01) for female neonates.

Values recorded for cord length with respect to central, eccentric and marginal cord insertions were $43.00 \mathrm{~cm}$ (SE 3.48), $38.30 \mathrm{~cm}$ (SE 2.63) and $35.67 \mathrm{~cm}$ (SE2.73) for females respectively and that of males were $39.40 \mathrm{~cm}$ (SE 1.87), 42.38 $\mathrm{cm}$ (SE 3.81) and $53.67 \mathrm{~cm}$ (SE 2.73) for central, eccentric and marginal respectively.

\section{DISCUSSION}

Analysis of the result obtained shows that the average birth weight of neonate was $3172.90 \mathrm{~g}$ (SE 107.96). This result concurs with the result obtained by Mondal [3], who reported that the average birth weight of neonate was $3200 \mathrm{~g}$. The value obtained in this study also shows that the average weight of the neonate was above the low birth weight (LBW) value defined by Bloomberg [5]. From table 1 the mean placental weight was 452.10g (SE 13.20) for the sample size of 40 neonates. From other studies such as that reported by Tsegah [17], it was recorded that the normal placental weight was $474.00 \mathrm{~g}$ (SE 14.23). The scatter plot (figure 9) of birth weight and placental weight shows a significant positive correlation $(r=0.478, p<0.002)$ between birth weight and placental weight. The result obtained shows that eccentric position of the cord on the placenta occurs in most of the neonatal delivery cases than the central and marginal positions. Out of the 40 samples, 23 eccentric positions (57.5\%), 11 central positions $(27.5 \%)$ and 6 marginal positions (15\%) were recorded. The predominance of eccentric position concurs with the report by Tsegah [17], Ahenkorah [19] and Addai [20]. The average neonatal weight for females was $2882.30 \mathrm{~g}$ (SE $189.07)$ and that of the male neonates was $3289.85 \mathrm{~g}$ (SE 191.92). This signifies that the occurrence of eccentric position produces a higher birth weight in male than in female neonates. It could be observed that centrally positioned cord on the placenta produces a larger neonatal weight for females $(3530.00 \mathrm{~g}$, SE 446.52) than its occurrence in males (3012.00g, SE 136.65). The result shows that marginal cord positions for both male and female with neonatal weight of 3108.33g, (SE 300.75) and 3253.33g (SE 249.69) respectively.

The total mean value for neonatal weight recorded for each cord position was not significantly difference from the other cord positions. This inference concurs with the report by Shun-Jen (2010) that the position of umbilical cord on the placental does not have much effect on the neonatal weight. The total mean weight of neonates for central umbilical cord position was $3294.55 \mathrm{~g}$ (SE 253.82), marginal umbilical cord position was $3180.83 \mathrm{~g}$ (SE 177.79) and eccentric umbilical cord position was $3180.83 \mathrm{~g}$ (SE 177.79). The summary in table 4 shows the mean placental weights for central, eccentric and marginal were $441.27 \mathrm{~g}$ (SE 23.21), $462.61 \mathrm{~g}$ (SE 15.99) and $431.67 \mathrm{~g}$ (SE $50.61)$ respectively. Again it could be deduced that although both male and females had $n=3$ for marginal cord insertion, the male had more variation of PW (SE 105.63) than in the females (SE 29.06).

For centrally positioned umbilical cord the total mean placental volume was $397.73 \mathrm{~cm} 3$ (SE 21.45), eccentric position was $410.13 \mathrm{~cm} 3$ (SE 18.54) and marginal position was $430.83 \mathrm{~cm} 3$ (58.86) with 441.27g (SE 23.21), 462.61g (SE 15.99 ) and $431.67 \mathrm{~g}$ (SE 50.61) respectively. This suggests that the volume of the placenta is not 
affected by the position of the umbilical cord. The mode of umbilical cord position for central cord position was $2.05 \mathrm{~cm}$ (SE 0.09), eccentric position was $1.86 \mathrm{~cm}$ (SE 0.07) and marginal position was $2.18 \mathrm{~cm}$ (SE 0.11) with diameters of $18.91 \mathrm{~cm}$ (SE 0.58), $19.83 \mathrm{~cm}$ (SE 0.36) and $19.17 \mathrm{~cm}$ (SE 1.19) respectively.

The results showed a positive correlation between placental index and umbilical cord length. The $r$ value shows a moderate correlation between placental index and neonatal weight and placental variables. Since the $P$-values are less than 0.05 , the correlations are significant.

\section{CONCLUSION}

From the study it was observed that most of the neonatal weights recorded from the Central Regional Hospital are within the normal range of birth weight. Analysis of correlation between neonatal weight (BW) and placental weight (PW) showed that there was positive correlation that is significant $(r=0.616, p<0.002)$ between the two variables (BW and $\mathrm{PW}$ ) for eccentric cord position ( $p<0.05$ ). Though eccentric cord position resulted in higher birth weight for males than for female neonates, and central cord position also gave higher birth weight in females than male neonates; none of these parameters were statistically significant.

We therefore conclude that the position of the umbilical cord may not significantly influence neonatal weight or placental variables.

\section{Conflicts of Interests: None}

\section{REFERENCES}

[1]. Sadler T. W. Langman's medical embryology, $7^{\text {th }}$ Edition Twin Bridges Publication, Montana, USA. 2008;352-355.

[2]. Schnieke, C., Prussian, K. H, Barksdale D. J. and Dieckman J. Racial and ethnic differences in the presentation of birth weight. J Nurse Pract. 2007;3:229-239.

[3]. Mondal, B. Low birth weight in relation to sex of baby, maternal age and parity: A hospital based study on Tangsa tribe from Arunachal Pradesh. J. Ind. Med. Assoc. 1998; 96(12):362-370.

[4]. Cruikshank, D.W. and Breech, A. Other Malpresentations, and Umbilical Cord Complications, in: Scott, J. R., et al. (eds.), Danforth's Obstetrics and Gynecology, 9th Edition. Philadelphia, Lippincott Williams and Wilkins, 2003;381-395
[5]. Bloomberg, J. H. Placental weight relative to birth weight and long-term cardiovascular mortality. American Journal of Epidemiology 2009;170 (5): 662.

[6]. Canola, H., Balkau, B., Charles, M. S. Comments on the provisional report from the WHO consultation. European Group for the Study of Birth weight. Pedi Med 1992; 16:442-3.

[7]. Shun-Jen, T., Chi-Huang, C., Gwo-Jang, W., Wei-Hwa, C. and Cheng-Chang, C. Fetal demise by umbilical cord around abdomen and stricture. Archives of Gynecology and Obstetrics 2010; 281(1):137-139.

[8]. Kent, G., and Kliman, H. J. The placenta revealed. American journal of pathology. 1993;143(2): 332336

[9]. Fox, H. and Uyanwah-Ak-pom, A. S. Pathology of the placenta.10 ${ }^{\text {th }}$ Edition. Sanders Pub., London. 1977;135.

[10]. Hamilton, D., Guimaraes, I.C. and Guimaraes, A.C. Prevalence of cardiovascular risk factors in selected samples of school children - socioeconomic influence. PrevCardiol 2005;8(1):23-28.

[11]. Glenister, T. W. A. and Jean, R. W. S. Anatomy and Physiology for Nurses. $3^{\text {rd }}$ Edition. New Delhi, India. CBS Publishers and Distribution. 2004;71, 77 and 204.

[12]. Monie, D. M., Margaret, A. C. and Anna, G.W. N. Myles Textbook for Midwives. $14^{\text {th }}$ Edition. Churchill Livingstone. China. P1997;763-778.

[13]. Salafia, M. S. and Vintzileos, D. F. Determinants of low birth weight. Bull. WHO 1987;65:663-737.

[14]. Herbez, M. N., Herbst-Damm, K. L. and Kulik, J. A. Volunteer support, marital status, and the survival times of terminally ill patients. Health Psychology, 2005;24:225-229.

[15]. Jauniaux, S., Onie, G. A. and Katz, M. J. Umbilical Cord Complications, Wild Iris Medical Education 2007, Inc. NURSING CEU.com. In www.acc.unsystem.org/scn

[16]. Mayhew, R. The human placenta. $6^{\mathrm{TH}}$ Edition. Aspen Publishers Inc. U.S.A. 1983;1-119.

[17]. Tsegah, K. M. Variation of placental index with the volumetric composition of terminal villi in spontaneously delivered placentas (MPhil Thesis). Department of Anatomy, University of Ghana. 2009;1-94.

[18]. Sillick, T. J. and Schutte, N. S. Emotional, intelligence and self-esteem mediate between perceived early prenatal care and antenatal care. Applied Psychology, 2006;2(2):38-48.

[19]. Ahenkorah, J. Studies on morphological variations of the human umbilical cord (MPhil Thesis). Department of Anatomy, University of Ghana Medical School. 2003;1-80.

[20]. Addai, F.K., Ockleford, C. D. The mode of insertion of umbilical cord and vessels: association with maternal haemoglobin gynotype, neonatal factors, and placental components volumes. Anat. Embryol. 1994;189:107-114. 